\title{
Modification of the synchronous motor model for topological optimization
}

\author{
Timur Petrov ${ }^{1, *}$, and Alfred Safin ${ }^{1}$ \\ ${ }^{1}$ Kazan State Power Engineering University, Kazan, Russia
}

\begin{abstract}
Synchronous motors are increasingly used in industry, and they need to be made more energy efficient. To do this, it is possible to optimize the rotor of the synchronous motor by changing the design, obtaining the best option due to topological optimization. But the standard design of a synchronous motor is quite complicated for calculations by the finite element method, so it is necessary to simplify it as much as possible. To make all elements the simplest from the geometric point of view (rectangles, straight lines as much as possible, etc.), and to make for calculation not the complete model, but the smallest sector for which topological optimization can be adequately calculated. To do this, it is necessary to calculate the magnetic moment for a standard design, for each step of structural change, and compare these values. If there is a slight discrepancy in the data, it will be necessary to choose the sector correctly and confirm the adequacy of the model by comparison. All these calculations should be performed for the predicted model, which should be obtained after topological optimization.
\end{abstract}

\section{Introduction}

It has long been known that permanent magnet synchronous motors (PMSM) have better technical characteristics than conventional asynchronous motors, but from an economic point of view, they are much more expensive [1,2]. One reason is the high cost of rare earth magnets, which are used in permanent magnet synchronous motors [3]. Thus, if you use fewer magnets, then we can reduce the payback period, and make the use of synchronous motors more attractive.

According to the design of the rotor, synchronous motors can be classified into 2 types:

- surface permanent magnet synchronous motor (SPMSM);

- interior permanent magnet synchronous motor (IPMSM).

Built-in magnets can provide a higher torque value, but they are more difficult to manufacture, and the exact same number of magnets is used as for surface magnets $[4,5]$. However, it is possible to make the rotor in such a way that the magnets were located in it, not just in the form of trapezoid or rectangles, but of arbitrary shape, in order to reduce the number of magnets. And in order to determine the optimal design of the rotor, it is necessary to use topological optimization, and iterate over the options not just by enumeration, but by a genetic algorithm [6]. However, if you try to optimize the standard design of the synchronous motor, then there will be difficulties with the number of finite elements, and it is necessary to simplify the design element as much as possible, which will be shown in this article.

\section{Initial data}

For optimization, the engine was selected 11 DVM (valve engine), hereinafter referred to as the "engine".

The engine refers to general-purpose products, both continuous and cyclic, non-repairable. The engine is designed to work with a special semiconductor converter (electric drive).

This machine has the following characteristics:

- shaft rotation frequency of $750 \mathrm{rpm}$;

- voltage in the DC link of the electric drive $520 \mathrm{~V}$;

- without rotor position sensor, climatic modification B2;

- rated power $15 \mathrm{~kW}$;

- long-term permissible motor current at a moment of load on the shaft - $40 \mathrm{~A}$;

-lonely permissible moment of load on the shaft at a speed of $100 \mathrm{Nm}$;

- operating ambient temperature from minus 60 to plus 50;

- relative humidity of the environment of $80 \%$ at a temperature of plus 27 ;

- degree of protection of the IP55 engine.

According to the number of permissible operable states, engines belong to products of type 1, which during operation can be either in an operable state (state with nominal efficiency) or in an inoperable state (state of failure) [7].

Depending on the application mode, the engines belong to equipment operating in continuous use mode and the main part of the operating time is in operation mode $[8,9]$.

* Corresponding author: tobac15@mail.ru 
We have chosen this engine, since it is a rather simple design for topological optimization, and already based on the algorithm for our PMSM we will develop a universal optimization program.

To check the optimization, we will use the ELCUT program. ELCUT is a modern program for engineering modelling of electromagnetic, thermal and mechanical problems by the finite element method, which is suitable for calculating torque. ELCUT contains a magnetostatics module that can be used to calculate and analyse devices such as a solenoid, electric machines, magnetic screens, permanent magnets, magnetic disks, and the like.

\section{Model modification}

An example of calculating the magnetic induction for an engine is presented in Figure 1, these data agree with the values that we obtained during the tests.

The grooves were set as follows $\mathrm{A}+\mathrm{A}+\mathrm{B}-\mathrm{B}-\mathrm{C}+\mathrm{C}$ $+\mathrm{A}-\mathrm{A}-\mathrm{B}+\mathrm{B}+\mathrm{C}-\mathrm{C}-$, the current density was calculated based on the current and the area of the groove, and amounted to $7 \mathrm{~A} / \mathrm{mm} 2$.

The magnets are made of neodymium, and the direction of the coercive force is selected based on the location of the magnet, the stator, concentrator and air are set by standard values for magnetostatics tasks.

For the same example, we can get the value of the torque, however, for the optimization to be correct, we need to get the moment for the engine to rotate 90 degrees, and for these parameters calculate the root mean square moment. The Labelmover subroutine allows you to rotate the engine, in our case it is 18 steps of 5 degrees.

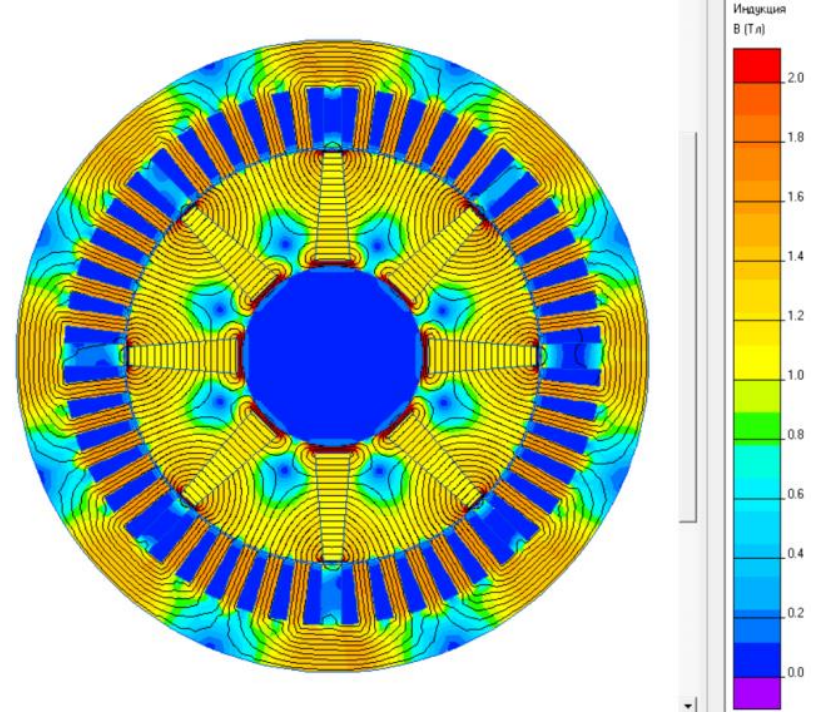

Fig. 1. Magnetic induction motor.

Modification of the synchronous motor model will be as follows:

1. Change the design of the grooves. Let's move from the complex design of the grooves to the simplest in terms of geometry (Figure 2). The difference between the moments of the first and last option is $9 \%$, and the form of the graph of the change in the moment in time is preserved, so that it can be argued that this simplification can be used.

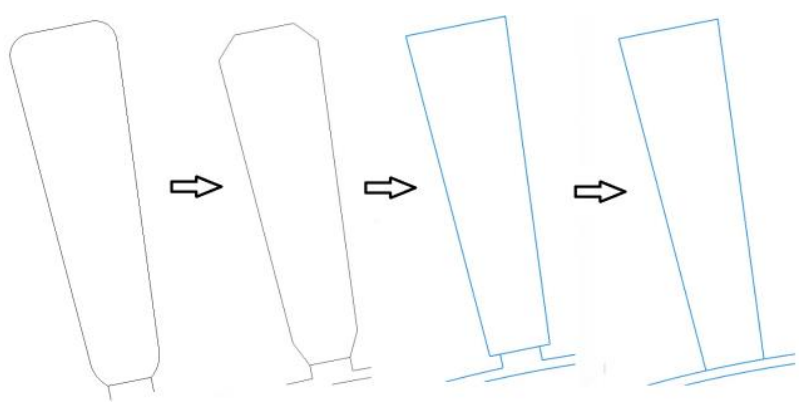

Fig. 2. Changing the design of the groove.

2. In the ELCUT program, the most accurate torque is calculated using the automatic grid sampling step, and thus for the engine the number of grid elements is about 100,000 , which is impossible to calculate topological optimization. Therefore, it is considered possible to make a grid with a manual sampling step of $2.5 \mathrm{~mm}$, the shape of the graph of the change in time is also saved.

It is important to clarify that during optimization we will compare a specific design and design after optimization; therefore, the accuracy of the obtained torque is not important to us, but an increase in its value is important.

3 . Given the symmetry of the engine, you can take half the engine, and check whether the torque decreases by about half. In the calculation, we got a decrease of 2.2 times, and maintaining the shape of the graph (Figure 3), and based on the shape of the magnetic field, we came to the conclusion that you can use the sector at 45 degrees.

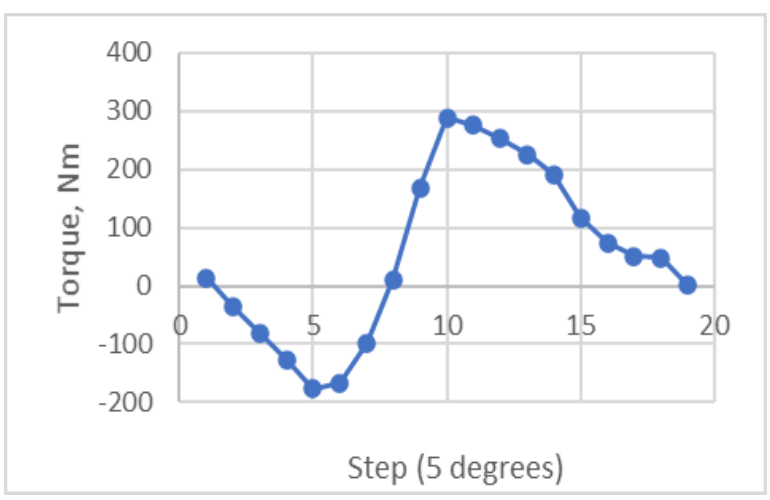

Fig. 3. Engine sector torque.

\section{Actions to Improve Optimization Efficiency}

First you need to take cells of $5 \mathrm{~mm}$ each, select local maxima from them (you cannot compare them at this stage, just collect the set). We can say that we have a rms moment of more than $10 \mathrm{Nm}$ for example - these are all local maxima. And then, based on these local maxima, carry out the calculation, reducing the cell size. But in general, anyway, one local maximum will be obtained with one version and the geometry of the windings $[10,11]$. 
Accordingly, the problem can be rephrased: not searching for the best option, but searching for local maxima, because at this stage we will not find the best option. Cells should be taken at $3 \mathrm{~mm}$ for optimal construction. Anything more than $50 \mathrm{Nm}$ is acceptable we assemble into a set.

Then, at the next stage, these local maxima can be clustered by neural networks. And then on clustered to conduct in-depth study. Or manually cluster, but it will be too time-consuming task.

And for the most effective solution, it is necessary to divide each square diagonally in both directions and sort through both options for dividing each square with a diagonal. So, an even shape can be obtained, but not square faces, because of which the moment can be considered 2 times more or less [12].

And the turn can be removed and collect everything that at the 10th step more than $50 \mathrm{Nm}$ gives the moment, so reduce the search time [13]. And plus, we use sectors of 22.5 degrees (easier calculation). If we perform all these operations, we can also count for $3 \mathrm{~mm}$ cells.

And it will also be necessary to carry out cleaning, it looks as follows:

We check the cell, if the cell above and below and left and right are not equal to the value of this cell, then the cell takes such a value, which are larger around. If all the cells around are different, then any value presented is accepted [14].

It is also important to consider that this optimization will not contain cells with air material for ease of calculation, however, it is obvious that this is impractical, therefore this point will be taken into account in the subsequent optimization of thermal characteristics, though also topological optimization. And in the end, a strength calculation will be performed to verify the resulting design [15].

\section{Conclusion}

Topological optimization provides the concept of a magnetic system, i.e. a rough picture of the arrangement of magnets. And then you can optimize the design by varying the width and height of the magnets in order to achieve the practical implementation of the rotor design.

This article demonstrates how you can go from the complex standard design of a permanent magnet synchronous motor to the simplest one (sector - 45 degrees, shown in Figure 4).

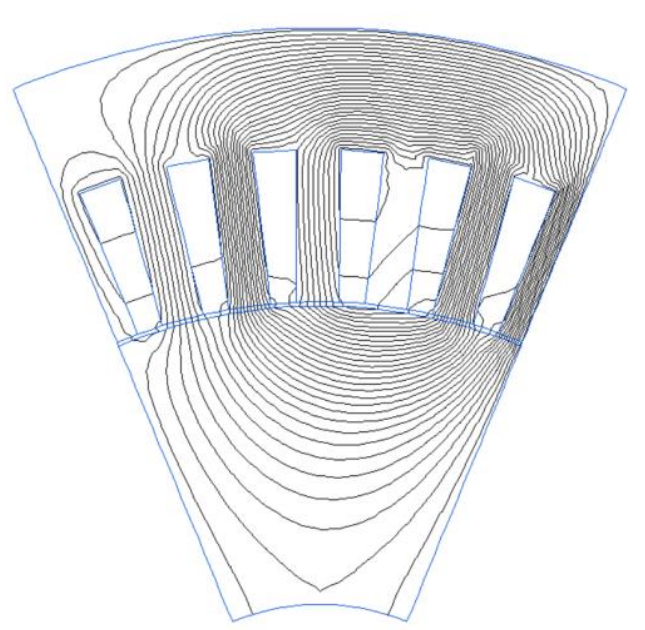

Fig. 4. The final version of the sector for calculation.

While the adequacy of the model remains at a high level, and with this model it is possible to carry out topological optimization through genetic an algorithm. The speed of calculations will increase with the help of computer software more than 10 times.

\section{Acknowledgments}

The reported study was funded by RFBR, project number 1937-90134.

\section{References}

[1] B.E. Vasiliev, L.A. Magerramova, Analysis of the possibility of using topological optimization in the design of uncooled turbine blades, Vestnik SGAU 3-1 (2015)

[2] C. Li, I.Y. Kim, Multi-material topology optimization for automotive design problems, Proceedings of the Institution of Mechanical Engineers, Part D: Journal of Automobile Engineering 232, 14, 1950-1969 (2018)

[3] E.I. Gracheva, O.V. Fedorov, Forecasting Reliability Electrotechnical Complexes of InPlant Electric Power Supply Taking into Account Low-Voltage Electrical Apparatuses, 2019 International Conference on Industrial Engineering, Applications and Manufacturing (ICIEAM), Sochi, Russia, 1-5 (2019)

[4] K.A. Bashin, R.A. Torsunov, S.V. Semenov, Methods of topological optimization of structures used in the aerospace industry, Bulletin of the PNRPU, Aerospace engineering 4, 51 (2017)

[5] K.L. Shenoy, M.S. Kumar, Design topology and electromagnetic field analysis of Permanent Magnet Brushless DC motor for electric scooter application, International Conference on Electrical, Electronics, and Optimization Techniques, ICEEOT 2016, 1541-1545 (2016)

[6] T.I. Petrov, A.R. Safin, I.V. Ivshin, A.N. Tsvetkov, V.Y. Kornilov, The Prospects of Using a Synchronous Machine with Permanent 
Magnets in the Oil Industry, 14th International Scientific-Technical Conference on Actual Problems of Electronic Instrument Engineering, APEIE 2018 - Proceedings, article no. 8546157, 336-338 (2018)

[7] H. Shim, S. Wang, K. Hameyer, Topology optimization of magneto thermal systems considering eddy current as Joule heat, IEEE Trans. Magn. 43, 4, 1617-1620 (2007)

[8] D.N. Dyck, D.A. Lowther, Automated design of magnetic devices by optimizing material distribution, IEEE Trans. Magn. 32, 3, 11881193 (1996)

[9] S. Wang, D. Youn, H. Moon, J. Kang, Topology optimization of electromagnetic systems considering magnetization direction, IEEE Trans. Magn. 41, 5, 1808-1811 (2005)

[10] A. Safin, A. Kopylov, R. Gibadullin, T. Petrov, R. Khusnutdinov, O. Fedorov, Thermal Model of a Linear Electric Machine, 1st International Conference on Control Systems, Mathematical Modelling, Automation and Energy Efficiency (SUMMA), Lipetsk, Russia, 426-428 (2019)

[11] D.H. Kim, J.K. Sykulski, D.A. Lowther, The implications of the use of composite material in electromagnetic device topology and shape optimization, IEEE Trans. Magn. 45, 3, 11541157 (2009)

[12] J. Lee, N. Kikuchi, Structural topology optimization of electrical machinery to maximize stiffness with body force distribution, IEEE Trans. Magn. 46, 10, 3790-3794 (2010)

[13] E.I. Gracheva, O.V. Naumov, A.N. Gorlov, Modelling Characteristics of Reliability LowVoltage Switching Devices on the Basis of Random Checks on the Example of Contactors, Proceedings - 2019 1st International Conference on Control Systems, Mathematical Modelling, Automation and Energy Efficiency, SUMMA 2019, article no. 8947595, 641-643 (2019)

[14] J.S. Choi, J. Yoo, Structural topology optimization of magnetic actuators using Genetic algorithms and ON/OFF sensitivity, IEEE Trans. Magn. 45, 5, 2276-2279 (2009)

[15] L.L. Myagkov, S.M. Sivachev, E.E. Strizhov, S.P. Chirsky, Topological optimization of a highperformance diesel piston, Dvigatelestroyeniye, 2, 3-10 (2018) 